\title{
SIMULATION STUDY OF ELECTRONIC DAMPING OF MICROPHONIC VIBRATIONS IN SUPERCONDUCTING CAVITIES*
}

\author{
Alicia Hofler ${ }^{\#}$ and Jean Delayen ${ }^{\dagger}$ \\ Thomas Jefferson National Accelerator Facility, Newport News, VA 23606, U.S.A.
}

\begin{abstract}
Electronic damping of microphonic vibrations in superconducting rf cavities involves an active modulation of the cavity field amplitude in order to induce ponderomotive forces that counteract the effect of ambient vibrations on the cavity frequency. In lightly beam loaded cavities, a reduction of the microphonicsinduced frequency excursions leads directly to a reduction of the rf power required for phase and amplitude stabilization. Jefferson Lab is investigating such an electronic damping scheme that could be applied to the JLab $12 \mathrm{GeV}$ upgrade, the RIA driver, and possibly to energy-recovering superconducting linacs. This paper discusses a model and presents simulation results for electronic damping of microphonic vibrations.
\end{abstract}

\section{INTRODUCTION}

One of the early challenges in the application of $\mathrm{rf}$ superconductivity to particle accelerators, especially ion accelerators, was the control and stabilization of the phase and amplitude of the accelerating fields in the large number of independent cavities. Ambient noise and microphonics can cause frequency variations that are larger than the bandwidth of the resonators.

Historically, high-velocity superconducting resonators have been used in high-current applications where most of the rf power was transferred to the beam. Recently, however, high-velocity elliptical-type resonators will be used in applications where the beam loading will be small and the need for phase and amplitude control will dictate most or all the requirements for $\mathrm{rf}$ power.

In the case of no beam loading, the minimum amount of rf power required for phase stabilization by negative feedback is given by $P=U \delta \omega$ [1-3], where $U$ is the energy content at operating gradient and $\delta \omega$ is the maximum amount of detuning at which phase lock is to be maintained. $\delta \omega$ has two components: a static component given by the accuracy with which the average cavity resonance frequency can be matched to the master reference frequency and a dynamic component due to microphonics-induced frequency excursions. With a welldesigned mechanical tuning system, the static component can be made much smaller than the dynamic component, and any reduction of the microphonics would lead to a corresponding reduction in the rf power requirements.

Mechanical stiffening of resonators is often used to reduce the Lorentz detuning and may also reduce, to some

\footnotetext{
* This work was supported by the U.S. DOE Contract No DE-AC0584-ER40150.

"hofler@jlab.org, ’delayen@jlab.org
}

extent, the microphonics. Mechanical damping has been very effective in some low-velocity structures [5], but has not yet been implemented in elliptical-type cavities. Electronic damping has also been used in low-velocity structures [2]; in this paper, we present an analytical study of electronic damping as a possible means of reducing microphonics in superconducting cavities.

\section{MODEL AND EQUATIONS}

We will use the same model described in more details in [1-4], namely a resonator operated in a self-excited loop. Since microphonics damping would be useful only in cavities that are lightly beam-loaded, we have ignored the influence of the beam current in the present study. In [1] it was found advantageous to operate the loop slightly off resonance on the low frequency side $\left(\theta_{l}<0\right)$; this introduced a small amount of coupling between phase and amplitude feedback which could be used to damp the microphonics. In [3] a feedback phase shifter $\left(\theta_{f}\right)$ was added that could be used to provide the same amount of coupling while still operating the unlocked self-excited loop on resonance $\left(\theta_{l}=0\right)$.

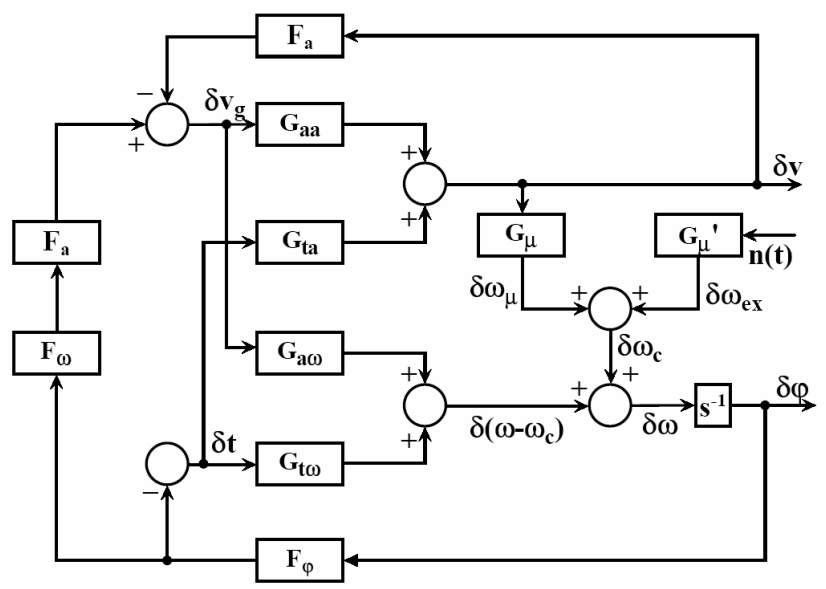

Figure 1: Transfer function representation of the system.

A block diagram of such a configuration can be found in $[3,4]$ and a transfer function representation of the system is shown in Fig. 1 where:

$$
\begin{aligned}
& G_{a a}=\frac{\cos \left(\theta_{l}+\theta_{f}\right)}{\cos \theta_{l}} \frac{1}{1+\tau s}, \quad G_{t a}=-\frac{\sin \left(\theta_{l}+\theta_{f}\right)}{\cos \theta_{l}} \frac{1}{1+\tau s}, \\
& G_{a \omega}=\frac{1}{\tau} \frac{\cos \left(\theta_{l}+\theta_{f}\right)}{\cos \theta_{l}}\left[y-\frac{y_{r}}{1+\tau s}\right], G_{t \omega}=\frac{1}{\tau} \frac{\cos \left(\theta_{l}+\theta_{f}\right)}{\cos \theta_{l}}\left[1+\frac{y y_{r}}{1+\tau s}\right], \\
& G_{\mu}=-\frac{2 \Omega_{\mu}^{2} k_{\mu} V_{0}^{2}}{s^{2}+\frac{2}{\tau_{\mu}} s+\Omega_{\mu}^{2}}, \quad G_{\mu}^{\prime}=\frac{\Omega_{\mu}^{2}}{s^{2}+\frac{2}{\tau_{\mu}} s+\Omega_{\mu}^{2}},
\end{aligned}
$$


$F_{a}, F_{\varphi}, F_{\omega}:$ Amplitude, Phase, and Frequency Feedbacks, $\tau=\frac{\tau_{0}}{1+\beta}:$ Loaded amplitude decay time, $y=\tan \left(\theta_{l}+\theta_{f}\right), y_{r}=\tau_{0}\left(\omega_{r}-\omega_{c}\right)(1+\beta)^{-1}=\tau\left(\omega_{r}-\omega_{c}\right)$

$G_{\mu}$ represents the coupling between the field amplitude and cavity frequency which is responsible for the ponderomotive instabilities [1], and $G_{\mu}^{\prime}$ represents the coupling between external sources and cavity frequency. $\Omega_{\mu}$ is the frequency of the mechanical mode of the cavity, and $\tau_{\mu}$ is its decay time.

The residual amplitude and phase errors due to fluctuations of the cavity eigenfrequency $\left(\delta \omega_{e x}\right)$ are

$$
\begin{gathered}
\delta v=-\delta \omega_{e x} F_{\varphi}\left(G_{t a}-G_{a a} F_{a} F_{\omega}\right) D^{-1}, \\
\delta \varphi=\delta \omega_{e x}\left(1+F_{a} G_{a a}\right) D^{-1},
\end{gathered}
$$

with

$$
\begin{array}{r}
D=\left[s+F_{\varphi}\left(G_{t \omega}-F_{a} F_{\omega} G_{a \omega}\right)\right]\left(1+F_{a} G_{a a}\right) \\
+F_{\varphi}\left(G_{\mu}-F_{a} G_{a \omega}\right)\left(G_{i a}-F_{a} F_{\omega}\right)
\end{array},
$$

and $\delta \omega_{e x}=G_{\mu}^{\prime} n(t)$, where $n(t)$ represents the external source of vibration.

\section{PERFORMANCE OF STABILIZATION SYSTEM}

If we assume that the fluctuations in resonator field phase and amplitude are due to fluctuations in cavity eigenfrequency, and that these in turn are due to the excitation of the mechanical mode by white noise of spectral density $A^{2}$, then the mean square values for the cavity frequency, and field amplitude and phase are given by [1]:

$$
\begin{aligned}
& <\delta \omega_{e x}^{2}>=A^{2} \int_{-\infty}^{+\infty}\left|-\omega^{2}+\frac{2}{\tau_{\mu}} i \omega+\Omega_{\mu}^{2}\right|^{-2} d \omega=A^{2} \frac{\pi \tau_{\mu}}{2 \Omega_{\mu}^{2}} \\
& <\delta v^{2}>=<\delta \omega_{e x}^{2}>\frac{2 \Omega_{\mu}^{2}}{\pi \tau_{\mu}} \int_{-\infty}^{+\infty}\left|\frac{G_{a}(i \omega)}{-\omega^{2}+\frac{2}{\tau_{\mu}} i \omega+\Omega_{\mu}^{2}}\right|^{2} d \omega \\
& <\delta \varphi^{2}>=<\delta \omega_{e x}^{2}>\frac{2 \Omega_{\mu}^{2}}{\pi \tau_{\mu}} \int_{-\infty}^{+\infty}\left|\frac{G_{\varphi}(i \omega)}{-\omega^{2}+\frac{2}{\tau_{\mu}} i \omega+\Omega_{\mu}^{2}}\right|^{2} d \omega
\end{aligned}
$$

where $G_{a}=-F_{\varphi}\left(G_{t a}-G_{a a} F_{a} F_{\omega}\right) D^{-1}$ and $G_{\varphi}=\left(1+F_{a} G_{a a}\right) D^{-1}$

The mean square errors $\left\langle\delta v^{2}\right\rangle$ and $<\delta \varphi^{2}>$ can be calculated in the most general case, but we will present the results under the following assumptions: no beam loading, loop phase adjusted so the unlocked cavity operates on resonance $\left(\theta_{l}=0\right)$, small feedback angle $\left(\theta_{f} \ll 1\right)$, large proportional feedback gains $\left(k_{a}, k_{\varphi} \gg 1, \tau \Omega_{\mu}\right)$, and $\tau / \tau_{\mu} \ll 1$.

\section{DAMPING BY FEEDBACK PHASE SHIFT}

As shown in $[3,4]$, the introduction of a feedback phase shifter $\theta_{f}$ introduces a coupling between the phase and amplitude feedback that can reduce the phase error at the expense of introducing an amplitude error. The rms phase and amplitude error are calculated to be [4]

$$
\begin{gathered}
<\delta \varphi^{2}>=\frac{\tau^{2}<\delta \omega_{e x}^{2}>}{k_{\varphi}^{2}}\left[1+\theta_{f} k_{\mu} V_{0}^{2} \frac{2 \tau}{k_{a}+1}\left(1-\frac{\tau_{\mu}}{2 \tau} \tau^{2} \Omega_{\mu}^{2} \frac{k_{\varphi}+k_{a}+1}{k_{\varphi}\left(k_{a}+1\right)}\right)\right] \\
<\delta v^{2}>=\frac{\tau^{2}<\delta \omega_{e x}^{2}>}{\left(k_{a}+1\right)^{2}}\left[\theta_{f}\right]^{2}
\end{gathered}
$$

\section{DAMPING BY FREQUENCY FEEDBACK}

The effectiveness of microphonics damping as described in the previous section depends on the amount of amplitude feedback and is reduced as the feedback gain is increased. A more effective way to damp microphonics would be to intentionally modulate the amplitude reference by an amount dependent on the instantaneous frequency offset between the cavity and the master reference with the appropriate phase shift in order to act as a damping mechanism.

As shown in [3], in the absence of beam loading and with no feedback phase shift $\left(\theta_{f}=0\right)$, the signal driving the resonator is of the form $V_{g}=V_{g o}\left[1+\delta v_{g}+i \delta t\right]$, where $\delta v_{g}=-F_{a} \delta v=-F_{a}(V-\mathrm{E}) / E$, and $\mathrm{E}$ is the amplitude reference. A modulation of the amplitude reference: $\mathrm{E}=\mathrm{E}_{0}(1+\delta e)$ introduces an additional term in the signal driving the resonator:

$$
\delta v_{g}(s)=-F_{a} \delta v(s)+F_{a} \delta e(s) .
$$

When the phase feedback gain $\left(k_{\varphi}\right)$ is sufficiently high, the "in quadrature" feedback signal $\delta t$ is directly proportional to the instantaneous phase error which, in turn, is proportional to the instantaneous difference between cavity and reference frequency. Thus $\delta t$ is an appropriate signal to provide a modulation of the amplitude reference: $\delta e(s)=-F_{\omega} \delta t(s)=\delta \varphi(s) F_{\varphi} F_{\omega}$, where $F_{\omega}$ is the frequency feedback transfer function.

In order to be effective as a damping mechanism, the frequency feedback needs to introduce a $\pi / 2$ phase shift between the frequency error and the amplitude modulation. For this reason, a good choice for $F_{\omega}$ is an integral-type feedback of the form:

$$
F_{\omega}=-k_{\omega} \frac{\Omega_{\mu}}{s} .
$$

The mean square frequency, phase, and amplitude errors can be calculated [4] and are:

$$
\begin{aligned}
& <\delta \omega_{\mathrm{c}}^{2}>=<\delta \omega_{e x}^{2}>\frac{1}{1+k_{\omega} \tau \tau_{\mu} \Omega_{\mu} k_{\mu} V_{o}^{2}} \\
& <\delta \varphi^{2}>=\frac{\tau^{2}<\delta \omega_{e x}^{2}>}{k_{\varphi}^{2}} \frac{1}{1+k_{\omega} \tau \tau_{\mu} \Omega_{\mu} k_{\mu} V_{o}^{2}} \\
& <\delta v^{2}>=\tau^{2}<\delta \omega_{e x}^{2}>\frac{k_{\omega}^{2}}{1+k_{\omega} \tau \tau_{\mu} \Omega_{\mu} k_{\mu} V_{o}^{2}}
\end{aligned}
$$




\section{SIMULATION RESULTS}

A Matlab model based on the transfer function representation in Fig. 1 has been developed for a JLab 12 $\mathrm{GeV}$ upgrade cavity operating in a self-excited loop at $20 \mathrm{MV} / \mathrm{m}$. This linear model has been used to compare the two damping schemes described in this paper. Both schemes can be useful at damping microphonics but the simulations confirm the analytical results that frequency feedback is the more effective of the two at reducing the cavity phase error while introducing smaller amplitude errors.

The simulation results shown in Figs. 2 and 3 assume a cavity operating at $20 \mathrm{MV} / \mathrm{m}$ with an electromagnetic coupling constant $k_{\mu}=3.69 \mathrm{~Hz} /(\mathrm{MV} / \mathrm{m})^{2}$ and a loaded quality factor $\mathrm{Q}_{\mathrm{L}}=3 \times 10^{7}$, and with a single mechanical mode at $\Omega_{\mu} / 2 \pi=34 \mathrm{~Hz}$ and decay time $\tau_{\mu}=300 \mathrm{msec}$. The amplitude and phase controllers are purely proportional and have fixed gains of 100. The frequency feedback controller used in the simulations is $F_{\omega}=-k_{\omega} \Omega_{\mu}\left(s+\frac{\Omega_{\mu}}{10}\right)^{-1}$. The simulations show the residual phase and amplitude errors when the mechanical mode is driven by a sinusoidal driving term $n(t)$ whose frequency is varied between 25 and $45 \mathrm{~Hz}$; the amplitude of $\mathrm{n}(\mathrm{t})$ is chosen to cause microphonics of amplitude $\delta \omega_{e x} / 2 \pi=10 \mathrm{~Hz}$ at the frequency of the mechanical mode.

It is apparent from Figs. 2 and 3 that, for the assumed parameters, the frequency damping via frequency feedback is far more effective in reducing the phase error while producing a modest amplitude error.

\section{CONCLUSION}

Analytical and numerical models for studying electronic damping of microphonics in lightly beamloaded superconducting cavities have been developed. Simulations have demonstrated the effectiveness of damping by frequency feedback.

\section{REFERENCES}

[1] J. R. Delayen, "Phase and Amplitude Stabilization of Superconducting Resonators", Ph.D. thesis, California Institute of Technology, 1978.

[2] J. R. Delayen, G. J. Dick, and J. E. Mercereau, “ A Microprocessor-Based Feedback System for Phase and Amplitude Stabilization of Superconducting Resonators", Proc. PAC 77, p. 1759.

[3] J. R. Delayen, "Phase and Amplitude Stabilization of Beam-Loaded Superconducting Resonators", Proc. Linac 92, p. 371.

[4] J. R. Delayen, "Electronic Damping of Microphonics in Superconducting Cavities", Proc. PAC 01, p. 1146.

[5] A. Facco, V. Zviagintsev, "Mechanical Stabilization of Superconducting Quarter-Wave Resonators", Proc. PAC 97, p. 3084.
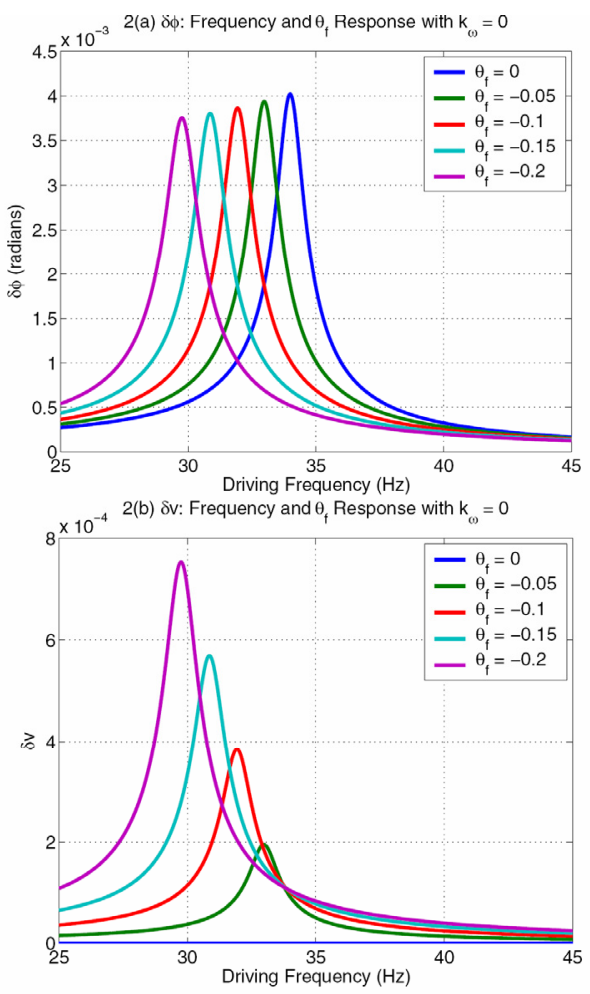

Fig.2: Residual phase (upper) and amplitude (lower) error as function of driving frequency for several feedback phase shifts.
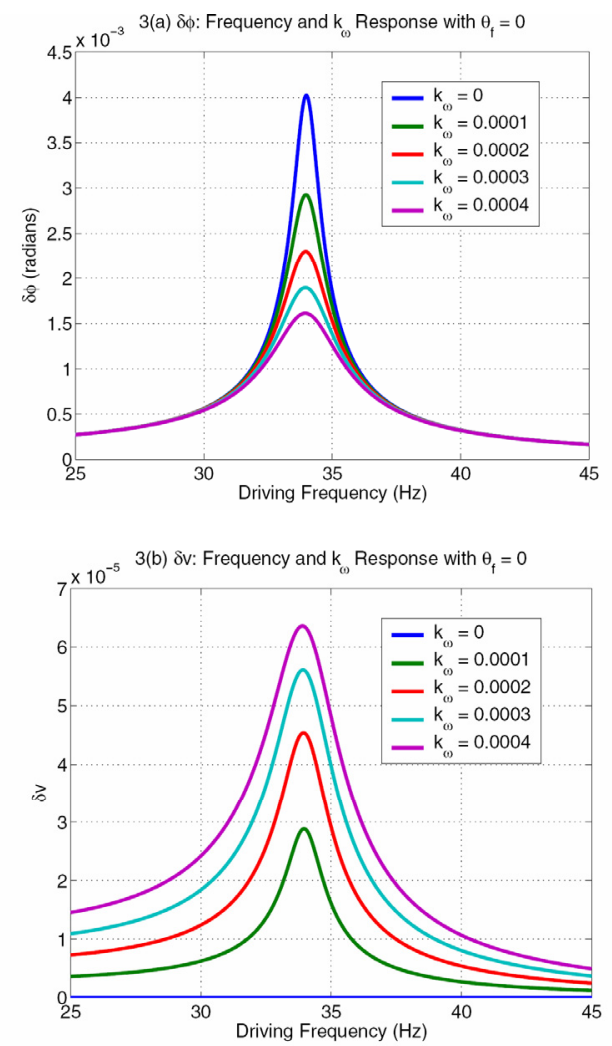

Fig.3: Residual phase (upper) and amplitude (lower) error as function of driving frequency for several frequency feedback gains. 